\title{
NLRC5 expression in tumors and its role as a negative prognostic indicator in stage III non-small-cell lung cancer patients
}

\author{
XIAOYU LI ${ }^{1}$, FUCHUN GUO ${ }^{1}$, YONGMEI LIU ${ }^{1}$, HUI-JIAO CHEN ${ }^{2}$, FENG WEN $^{1}$, BINWEN ZOU ${ }^{1}$,

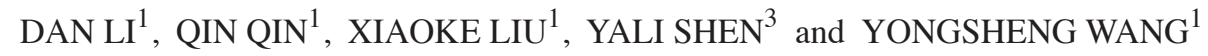 \\ ${ }^{1}$ Department of Thoracic Oncology, Cancer Center, State Key Laboratory of Biotherapy; ${ }^{2}$ Department of Pathology; \\ ${ }^{3}$ Department of Abdomen Oncology, Cancer Center, State Key Laboratory of Biotherapy, West China Hospital, \\ Sichuan University, Chengdu, Sichuan 610041, P.R. China
}

Received August 25, 2014; Accepted May 20, 2015

DOI: 10.3892/ol.2015.3471

\begin{abstract}
Major histocompatibility complex (MHC) class I molecules have a crucial role in tumor immune evasion; however, the association of MHC class I molecules with outcomes in cancer patients remains controversial. Nucleotide-binding oligomerization-like receptor family caspase recruitment domain-containing 5 (NLRC5) has been reported to be a MHC class I transactivator. However, the expression and function of NLRC5 in cancer remains to be elucidated. The present study aimed to retrospectively examine NLRC5 expression in human tumor tissues and its association with clinical outcomes of non-small-cell lung cancer (NSCLC) stage III patients. The expression of MHC class I and NLRC5 in NSCLC were detected using immunohistochemistry (IHC). The association between their expression levels was assessed using the Pearson's $\chi^{2}$ test and their association with survival was assessed using Kaplan-Meier analysis and the log-rank test. In addition, the expression of NLRC5 and MHC class I were examined in 323 cases of seven other types of tumors and their correlations were studied. The results revealed that the expression of NLRC5 was correlated with that of MHC class I in NSCLC patients ( $\mathrm{P}=0.008)$. MHC class I-positive and nuclear NLRC5-positive NSCLC patients were found to have shorter overall survival (OS) rates (log-rank, $\mathrm{P}=0.032$ and $\mathrm{P}=0.039$, respectively). In addition, in the seven different tumor types, there was a significant correlation between MHC class I and NLRC5 nuclear expression $(\mathrm{P}<0.001)$ as well as MHC class I and
\end{abstract}

Correspondence to: Professor Yongsheng Wang, Department of Thoracic Oncology, Cancer Center, State Key Laboratory of Biotherapy, West China Hospital, Sichuan University, 37 Guo Xue Xiang, Chengdu, Sichuan 610041, P.R. China

E-mail:wangys75@gmail.com

Key words: major histocompatibility complex class I, Nucleotide-binding oligomerization-like receptor family caspase recruitment domain-containing 5, tumor immune escape, non-smallcell lung carcinoma, prognosis indicators
NLRC5 cytoplasmic expression ( $\mathrm{P}=0.003)$. In conclusion, NLRC5 was demonstrated to be widely expressed in eight tumor tissues and its expression was correlated with that of MHC class I. Of note, nuclear NLRC5-negative and MHC class I-negative stage III NSCLC patients had improved OS rates compared to those with positive expression. Therefore, NLRC5 and MHC class I may be negative prognostic indicators in NSCLC stage III patients.

\section{Introduction}

Tumor immune evasion has a critical role in tumorigenesis and progression (1). Major histocompatibility complex (MHC) class I molecules are important for tumor immune evasion due to their function in antigen presentation to T-lymphocytes and cytotoxic T-lymphocytes (CTL) function (2). In addition, the loss of MHC class I is a common mechanism of experimental and spontaneous tumors, which allows them to evade recognition and destruction by CTLs $(3,4)$. In MHC class I downregulated or deficient tumor cells, tumor-associated antigens are not presented to the CTLs, which results in tumor immune evasion and affects the prognosis of tumor patients (3). However, whether MHC class I molecules have a positive or negative role in tumor patients' survival remains controversial. Certain studies have reported that MHC class I loss has poor outcomes due to its impact on antigen presentation to T-lymphocytes and CTLs $(5,6)$. By contrast, other reports have revealed that MHC class I loss may improve patients' survival through activating natural killer (NK) cell function $(7,8)$. These results vary between different types of tumors and the occurrence of lymph node metastasis $(5,7)$.

It has been reported that NLRC5 may have a positive role in the regulation of MHC class I expression in human cell lines and mice models. NLRC5 function was examined in NLRC5-deficient mice, the results of which revealed reduced MHC class I expression in lymphocytes, including T, NK and NKT lymphocytes $(9,10)$. NLRC5 localizes to the nucleus of lymphocytes, where it promotes MHC class I gene expression via stimulation of the H-2D and $\mathrm{H}-2 \mathrm{~K}$ gene promoters $(11,12)$. Human NLRC5 is predominantly expressed in hematopoietic cells as well as the spleen, lymph nodes, bone marrow 
and thymus; in addition, NLRC5 is expressed abundantly in the lungs and intestines (10,11,13-18). Staehli et al (10) reported that HeLa cells express NLRC5 when induced by interferon- $\gamma$. MHC class I molecules have a critical role in tumor immune evasion and are associated with cancer prognosis; since, NLRC5 was reported to act as a MHC class I transactivator, it may therefore affect tumor patients' survival through regulating tumor immune evasion through $\mathrm{MHC}$ class I. Thus, evaluating the expression of NLRC5 in human solid tumors and exploring its association with MHC class I expression and patients' survival may offer a novel method for predicting patient prognosis and provide potential novel therapeutic targets.

The present study aimed to examine NLRC5 expression in human tumor tissues and its association with clinical outcomes of non-small-cell lung cancer (NSCLC) stage III patients. The expression of NLRC5 and MHC class I was determined in non-small-cell lung cancer (NSCLC) stage III tissues and the correlation between NLRC5 (cytoplasmic and nuclear) and MHC class I was analyzed. In addition, the clinical data of NSCLC patients was collected in order to study the association between clinical outcomes and the expression of NLRC5 and MHC class I. Furthermore, the present study aimed to examine the expression of NLRC5 and MHC class I in seven different types of common human solid tumors in tissue microarrays (TMAs) using immunohistochemistry (IHC).

\section{Materials and methods}

NSCLC patients. A total of 62 NSCLC patients who underwent radical resection of stage III-node involvement $(\mathrm{N})_{2}$, without any preoperative therapy, were included in the present retrospective study. All patients were diagnosed and underwent surgery at West China Hospital (Chengdu, China) between January 2001 and September 2003. Histological diagnosis was established according to the guidelines of the World Health Organization (19). Pathological findings, including tumor size, location and lymph node status, were described in the reports of board-certified pathologists. Out of the 62 NSCLC patients, the pathological diagnoses included 31 with adenocarcinoma cell cancer, 24 with squamous cell cancer and 7 with other pathology types. Patients' age at the time of surgery ranged from 26 to 75 years, with a median age of 58 years. Long-term outcome was determined from hospital records and information from follow-up appointments. Overall survival (OS) was measured from the date of surgery to either mortality or the final follow-up visit. Progression-free survival was calculated from the date of surgery to the time of the first local or distant recurrence, or mortality from any cause. Local recurrence was defined as tumor regrowth in hilar, mediastinal, supraclavicular lymph nodes or at the bronchial margin of resection, as visualized using computed tomography (CT) scans. Recurrences beyond those sites were deemed as distant metastases. The present study was approved by the Ethics Committee of Sichuan University (Chengdu, China). Written informed consent was obtained from all patients and all clinical investigations were performed according to the principles of the Declaration of Helsinki.
TMAs. Seven different types of human tumor paraffin-tissues were purchased from the company of Ailina Biotechnology Co., Ltd (Xi'an, China), including 69 renal carcinoma cases (BC070140), 30 cervical carcinoma cases (CR602), 37 rectal cancer cases (RE482), 67 gastric adenocarcinoma cases (BS01012), 40 liver cancer cases (LV483), 40 malignant melanoma cases (ME418a) and 40 prostate cancer cases (PR483a). The TMAs were composed of normal tissue, adjacent tissue and different types of tumor tissues.

IHC analysis. NSCLC tissues were fixed in 10\% buffered formalin (Beyotime Institute of Biotechnology, Shanghai, China), embedded in paraffin (Beyotime Institute of Biotechnology) and cut into $5-\mu \mathrm{m}$ sections. TMAs and NSCLC sections were deparaffinized in xylene (Beyotime Institute of Biotechnology), rehydrated in a series of descending ethanol (Beyotime Institute of Biotechnology) concentrations and incubated in $0.03 \%$ hydrogen peroxide (Beyotime Institute of Biotechnology), then stored in dark place for $10 \mathrm{~min}$. Antigen retrieval was performed in $10 \mathrm{mM}$ sodium citrate buffer (pH 6.0; Beyotime Institute of Biotechnology) for $10 \mathrm{~min}$ at room temperature. The tissue sections and TMAs were then incubated with antibodies at room temperature for $45 \mathrm{~min}$. Commercial antibodies employed were rabbit monoclonal antibodies: Anti-NLRC5 (1:200; ab117624; Abcam, Cambridge, UK), anti-human leukocyte antigen (HLA)-ABC (1:100; ab70328; Abcam). Following incubation, the specimens were washed with Tris-buffered saline with Tween (TBS-T; 0.5\% Tween, $0.1 \mathrm{M}$ Tris-base, $0.9 \% \mathrm{NaCl}$; $\mathrm{pH}$ 7.6; Beyotime Institute of Biotechnology) and incubated with peroxidase-labeled polymer (Beyotime Institute of Biotechnology) at room temperature for $30 \mathrm{~min}$. The samples were then washed with TBS-T buffer and incubated with freshly prepared 3,3'-diaminobenzidine tetrahydrochloride (DAB; Zhongshan Jinqiao Biological Technology Ltd., Beijing, China) and substrate-chromogen buffer (Beyotime Institute of Biotechnology) at room temperature for $7 \mathrm{~min}$. Immunohistochemical reactions were developed in freshly prepared $\mathrm{DAB}$ at room temperature for $7 \mathrm{~min}$, then lightly counterstained with hematoxylin (Beyotime Institute of Biotechnology) prior to mounting. The intensity of staining and the percentage of positive cells were assessed in a semi-quantitative manner. Images were captured using an Olympus BX51 fluorescence microscope (Olympus Corp., Tokyo, Japan) equipped with an Olympus Micro DP 72 camera (Olympus Corp.). The distribution of positive cells was scored as follows: Not stained, 0 ; $<1 / 3$ cells stained, $1 ;<2 / 3$ cells stained, 2 ; and $>2 / 3$ cells stained, 3. The intensity of staining was graded as follows: Not stained, 0; mild stained, 1; and strong stained, 2. The scores for distribution and intensity were added and graded as follows: $0-2$, negative; and 3-5, positive (20).

Statistical analysis. Statistical analysis of the study data was performed using SPSS 17.0 for Windows (SPSS Inc., Chicago, IL, USA). The significance of associations was determined using the Pearson $\chi^{2}$ test. For OS analysis, Kaplan-Meier curves were derived and the statistical significance of differences between the survival of groups with different MHC class I and NLRC5 expression was determined using the log-rank test. The results were censored if the patients remained alive, had 
Table I. MHC class I and NLRC5 expression and association with OS in non-small-cell lung cancer.

\begin{tabular}{llccrr}
\hline Marker & Expression & $\mathrm{n}(\%)$ & Median OS (months) & $95 \% \mathrm{CI}$ & $\mathrm{P}$ \\
\hline MHC class I & Negative & $10(16.1)$ & 28.0 & $19.5-36.5$ & 0.032 \\
& Positive & $52(83.9)$ & 19.0 & $16.0-20.0$ & \\
CNLRC5 & Negative & $7(11.3)$ & 32.0 & $0.0-75.0$ & 0.086 \\
& Positive & $55(88.7)$ & 19.4 & $16.7-22.2$ & \\
NNLRC5 & Negative & $20(32.3)$ & 30.0 & $24.5-35.5$ & 0.039 \\
& Positive & $42(67.7)$ & 17.0 & $14.0-20.0$ & \\
\hline
\end{tabular}

OS, overall survival; CI, confidence interval; MHC, major histocompatability complex; C/NNLRC5, cytoplasmic/nuclear nucleotide-binding oligomerization-like receptor family caspase recruitment domain-containing 5.

Table II. Correlation of major histocompatibility complex class I and NLRC5 in seven common human solid tumors.

\begin{tabular}{lcccc}
\hline Tumor type & $\mathrm{n}$ & $\begin{array}{c}\text { HLA-ABC \& } \\
\text { NNLRC5 } \\
\text { P-value }\end{array}$ & $\begin{array}{c}\text { NNLRC5 \& } \\
\text { CNLRC5 } \\
\text { P-value }\end{array}$ & $\begin{array}{c}\text { HLA-ABC \& } \\
\text { CNLRC5 } \\
\text { P-value }\end{array}$ \\
\hline Renal carcinoma & 69 & 0.926 & 0.942 & 0.703 \\
Gastric adenocarcinoma & 67 & 0.014 & 0.010 & 0.045 \\
Cervical carcinoma & 30 & 0.295 & 0.363 & 0.885 \\
Prostate cancer & 39 & 0.018 & 0.746 & 0.342 \\
Malignant melanoma & 40 & 0.015 & 0.057 & 0.031 \\
Liver cancer & 40 & 0.008 & 0.002 & 0.570 \\
Rectal cancer & 37 & 0.019 & 0.812 & 0.354 \\
All tumor cases & 384 & $<0.001$ & 0.003 & $<0.001$ \\
\hline
\end{tabular}

(C/N)NLRC5, (cytoplasmic/nuclear) nucleotide-binding oligomerization-like receptor family caspase recruitment domain-containing 5; HLA-ABC, human leukocyte antigen-ABC.

died from any other causes or had withdrawn from the study. Cox regression analysis was used for multivariate analysis to allow for comparison of the effects of several different factors on survival. $\mathrm{P}<0.05$ was considered to indicate a statistically significant difference between values.

\section{Results}

Correlation of NLRC5 and MHC class I expression in NSCLC patients. NLRC5 and MHC class I expression were detected in 62 cases, including 31 with adenocarcinoma cell cancer, 24 with squamous cell cancer and 7 with other pathology types. IHC staining and analysis revealed that $83.9 \%$ of tissues exhibited MHC class I-positive expression, $67.7 \%$ of tissues demonstrated cytoplasmic NLRC5-positive expression and $88.7 \%$ were nuclear NLRC5-positive (Table I). The association study revealed that the expression of MHC class I was significantly correlated with the expression of nuclear NLRC5 ( $\mathrm{P}=0.008)$; in addition, nuclear NLRC5 was found to be associated with the expression of NLRC5 in the cytoplasm ( $\mathrm{P}=0.002)$. However, no correlation was observed between MHC class I and cytoplasmic NLRC5 ( $\mathrm{P}=0.570)$ (Table II). Fig. 1 shows representative IHC images of HLA-ABC, cytoplasmic and nuclear NLRC5 staining in NSCLC tissues, including negative cytoplasmic and nuclear NLRC5 staining, positive expression of NLRC5 in the cytoplasm and nucleus, HLA-ABC lose and HLA-ABC expression in the tumor cell membrane.

MHC class I and nuclear NLRC5 indicate the prognosis of NSCLC patients. Nuclear NLRC5 and MHC class I positive expression were revealed to be correlated with a reduced OS rate in NSCLC stage III patients (log-rank, $\mathrm{P}=0.039$ and $\mathrm{P}=0.032$, respectively) (Table I). The median OS of MHC class I-positive patients was 19 months, while the OS for MHC class I-negative patients was 28 months $(\mathrm{P}=0.032)$. The median OS of nuclear NLRC5-positive patients was 17 months, while the negative group was 30 months $(\mathrm{P}=0.039)$ (Fig. 2). For the cytoplasmic NLRC5-positive and -negative patients, the data were 32 months and 19.4 months, respectively; these results were not significantly associated with prognosis $(\mathrm{P}=0.086)$. By contrast, there was no significant association between the expression of MHC class I or NLRC5 and progression free survival (data not shown). Cox regression analysis was used for multivariate analysis to compare the effects of several different factors on survival, including gender, age, pathological type and tumor-node-metastasis values; however, no significant positive correlations were identified (Table III). 
Table III. Association between MHC class I, NLRC5 expressions and clinicopathologic factors in non-small-cell lung cancer patients.

\begin{tabular}{|c|c|c|c|c|c|c|c|}
\hline Characteristic & $\mathrm{n}$ & HLA-ABC+ $(\%)$ & $\mathrm{P}$ & NNLRC5+ (\%) & $\mathrm{P}$ & CNLRC5+ $(\%)$ & $\mathrm{P}$ \\
\hline Gender & & & 0.681 & & 0.349 & & 0.651 \\
\hline Male & 48 & $41(85.4)$ & & $34(70.8)$ & & 43 (89.6) & \\
\hline Female & 14 & 11 (78.6) & & $8(57.1)$ & & $12(85.7)$ & \\
\hline Age & & & 0.493 & & 1.00 & & 0.432 \\
\hline$>60$ & 33 & 29 (87.9) & & $22(66.7)$ & & $28(84.8)$ & \\
\hline$<60$ & 29 & $23(79.3)$ & & $20(69.0)$ & & $27(93.1)$ & \\
\hline \multicolumn{8}{|l|}{ Histological } \\
\hline $\mathrm{AC}$ & 31 & $24(77.4)$ & & $21(67.7)$ & & $26(83.9)$ & \\
\hline Classification & & & 0.359 & & & & 0.401 \\
\hline $\mathrm{SC}$ & 24 & 22 (91.7) & & $16(66.7)$ & & $22(91.7)$ & \\
\hline Others & 7 & $6(85.7)$ & & $5(71.4)$ & & $7(100.0)$ & \\
\hline TNM & & & 0.228 & & 0.625 & & 0.406 \\
\hline T1N2M0 & 3 & $2(66.7)$ & & $1(33.3)$ & & $2(66.7)$ & \\
\hline $\mathrm{T} 2 \mathrm{~N} 2 \mathrm{M} 0$ & 25 & $23(92.0)$ & & $17(68.0)$ & & $23(92.0)$ & \\
\hline T3N2M0 & 17 & 15 (88.2) & & 12 (70.6) & & $14(82.4)$ & \\
\hline T4N2M0 & 17 & 12 (70.6) & & 12 (70.6) & & $16(94.1)$ & \\
\hline
\end{tabular}

MHC, major histocompatability complex; (C/N)NLRC5, (cytoplasmic/nuclear) nucleotide-binding oligomerization-like receptor family caspase recruitment domain-containing 5; HLA-ABC, human leukocyte antigen-ABC; AC, adenocarcinoma; SC, squamous carcinoma; TNM, tumor-node-metastasis.
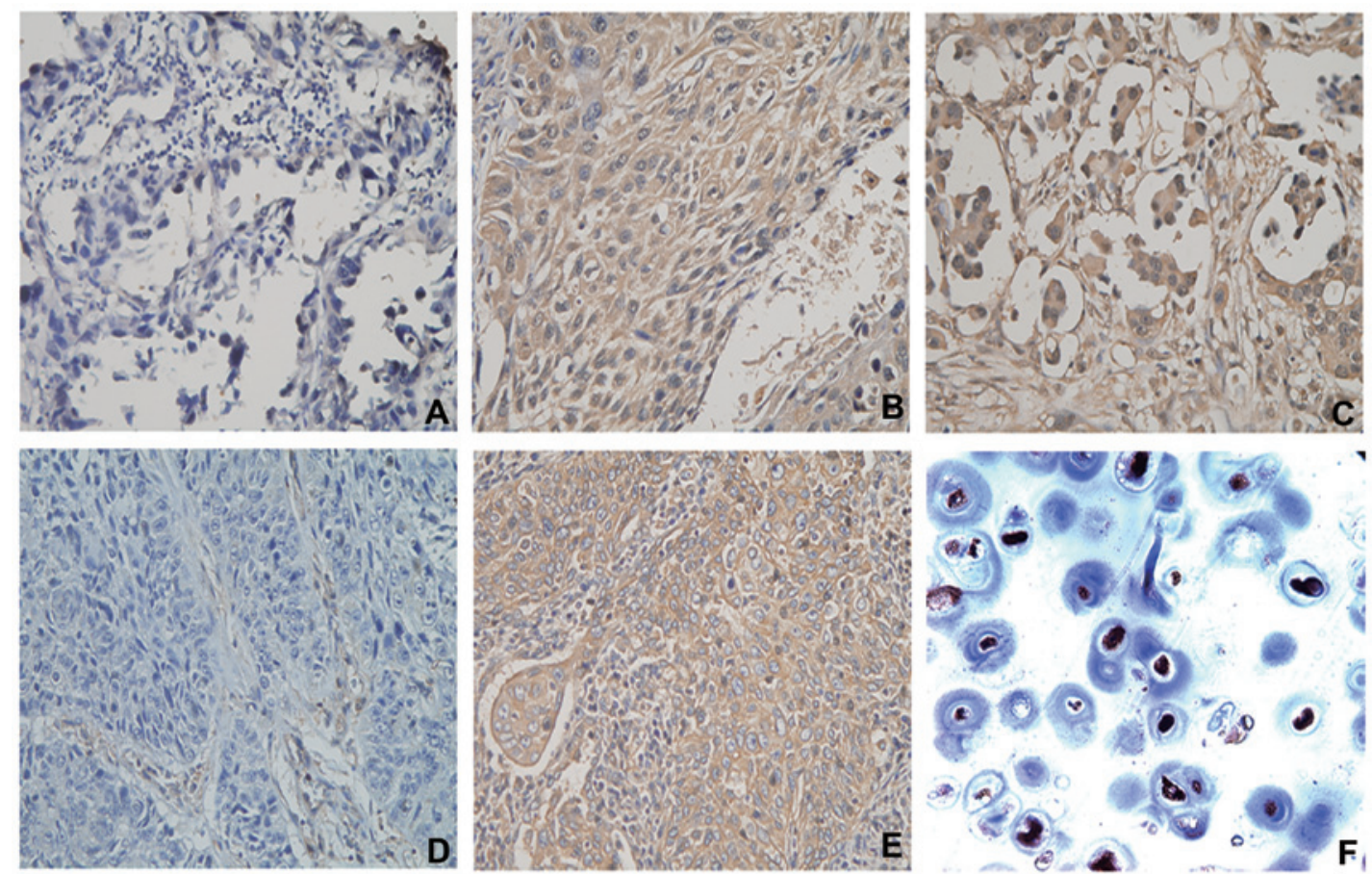

Figure 1. Immunohistochemical staining of HLA-ABC, cytoplasmic and nuclear NLRC5 in NSCLC tissues. Representative images of 62 cases of NSCLC with $\mathrm{N}_{2}$ nodal disease (magnification, $\mathrm{x} 400$ ). (A) Cytoplasmic and nuclear NLRC5-negative staining; (B) positive expression of NLRC5 in cytoplasm; (C) positive expression of NLRC5 in the nucleus; (D) HLA-ABC lose; (E) HLA-ABC expression in tumor cell membrane; and (F) positive control of NLRC5 in the human bronchus cartilage. HLA-ABC, human leukocyte antigen-ABC; NLRC5, nucleotide-binding oligomerization-like receptor family caspase recruitment domain-containing 5; NSCLC, non-small-cell lung cancer.

NLRC5 and MHC class I are expressed in different tumor tissues. The expression of NLRC5 and MHC class I were examined in 323 cases of tumor tissue in TMAs by IHC; the tumors investigated were the seven most common types of 
Table IV. Expression of major histocompatibility complex class I and NLRC5 in seven common human solid tumors.

\begin{tabular}{lcccr}
\hline Tumor type & $\mathrm{n}$ & HLA-ABC+ $(\%)$ & CNLRC5+ (\%) & NNLRC5+ (\%) \\
\hline Renal carcinoma & 69 & $57(87.6)$ & $61(88.4)$ & $51(73.9)$ \\
Gastric adenocarcinoma & 67 & $56(83.6)$ & $52(77.6)$ & $58(86.6)$ \\
Cervical carcinoma & 30 & $24(60.0)$ & $19(63.3)$ & $17(56.7)$ \\
Prostate cancer & 40 & $33(82.5)$ & $35(87.5)$ & $34(85.0)$ \\
Malignant melanoma & 40 & $27(67.5)$ & $30(75.0)$ & $27(67.5)$ \\
Liver cancer & 40 & $22(59.5)$ & $32(86.5)$ & $24(60.0)$ \\
Rectal cancer & 37 & & $32(83.8)$ & \\
\hline
\end{tabular}

(C/N)NLRC5, (cytoplasmic/nuclear) nucleotide-binding oligomerization-like receptor family caspase recruitment domain-containing 5; HLA-ABC, human leukocyte antigen-ABC.
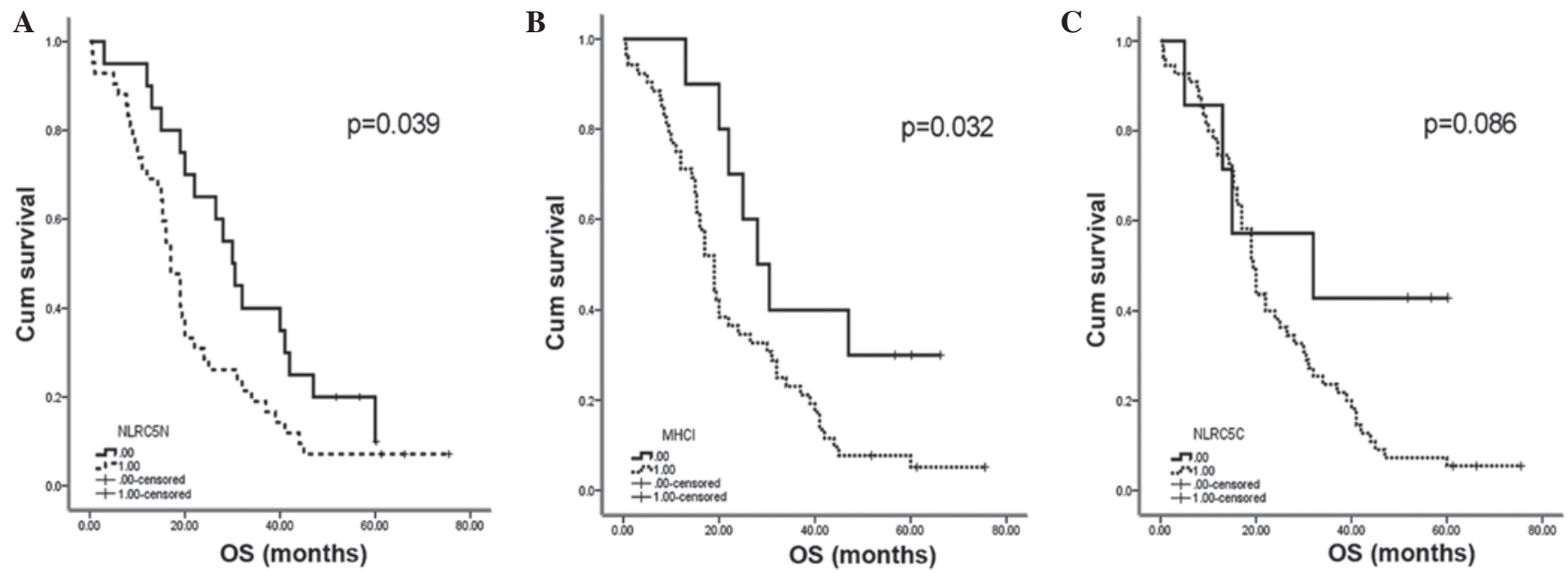

Figure 2. Kaplan-Meier survival analysis of OS in non-small-cell lung cancer patients in association with (A) human leukocyte antigen-ABC (B) nuclear NLRC5 and (C) cytoplasmic NLRC5. HLA-ABC and nuclear NLRC5 positive ones have a longer overall survival. MHCI, major histocompatability complex class I; (C/N)NLRC5, (cytoplasmic/nuclear) nucleotide-binding oligomerization-like receptor family caspase recruitment domain-containing 5.

human solid tumors. The results demonstrated that MHC class I was widely expressed in all seven types of tumor tissue examined and certain sections of the tissues demonstrated high levels of MHC class I expression. The percentages of MHC class I-positive TMAs were $82.6 \%$ (57/69) in renal carcinoma, $83.6 \%(56 / 67)$ in gastric adenocarcinoma, $60 \%$ $(18 / 30)$ in cervical squamous carcinoma tissue, $60 \%(24 / 40)$ in prostate cancer, $82.5 \%$ (33/40) in malignant melanoma, $67.5 \%(27 / 40)$ in liver cancer and $59.5 \%$ (22/37) in rectal cancer. For nuclear and cytoplasmic expression of NLRC5, there results were: $73.9 \%(51 / 69)$ and $88.4 \%(61 / 69)$ in renal carcinoma; $86.6 \%(58 / 67)$ and $77.6 \%(52 / 67)$ in gastric adenocarcinoma; $56.7 \%(17 / 30)$ and $63.3 \%(19 / 30)$ in cervical squamous carcinoma; $85.0 \%$ (34/40) and $87.5 \%$ $(35 / 40)$ in prostate cancer; $67.5 \%(27 / 40)$ and $75.0 \%(30 / 40)$ in malignant melanoma; 60\% (24/40) and $75.0 \%(30 / 40)$ in liver cancer; and $83.8 \%$ (31/37) and $86.5 \%$ (32/37) in rectal cancer, respectively (Table IV). Fig. 3 shows the immunohistochemical staining of seven human solid tumor tissue microarrays. Each panel shows a representative example of tumor tissue exhibiting positive HLA-ABC, cytoplasmic NLRC5 and nuclear NLRC5 staining.
Correlation of NLRC5 and MHC class I expression in tumors. In the 385 cases (62 NSCLC and 323 TMAs) of tumor paraffin-tissues for IHC analysis, the expression of MHC class I (HLA-ABC), cytoplasmic NLRC5 and nuclear NLRC5 were examined and their correlations were determined using the Pearson $\chi^{2}$ test. The results revealed significant correlations between MHC class I and nuclear NLRC5 $(\mathrm{P}<0.001)$, MHC class I and cytoplasmic NLRC5 $(\mathrm{P}=0.003)$ and between nuclear and cytoplasmic NLRC5 $(\mathrm{P}<0.001)$ (Table II). Furthermore, the correlations between the three proteins expression were analyzed in the seven TMAs separately. The results revealed that, with the exception of renal carcinoma and cervical cancer, all the tumor tissues demonstrated correlations between MHC class I and nuclear NLRC5 expression (Table II). These results indicated that, as a MHC class I transactivitor, the expression of NLRC5 was correlated with MHC class I in human solid tumors.

\section{Discussion}

To the best of our knowledge, the present study was the first to examine the expression of NLRC5 in common human 

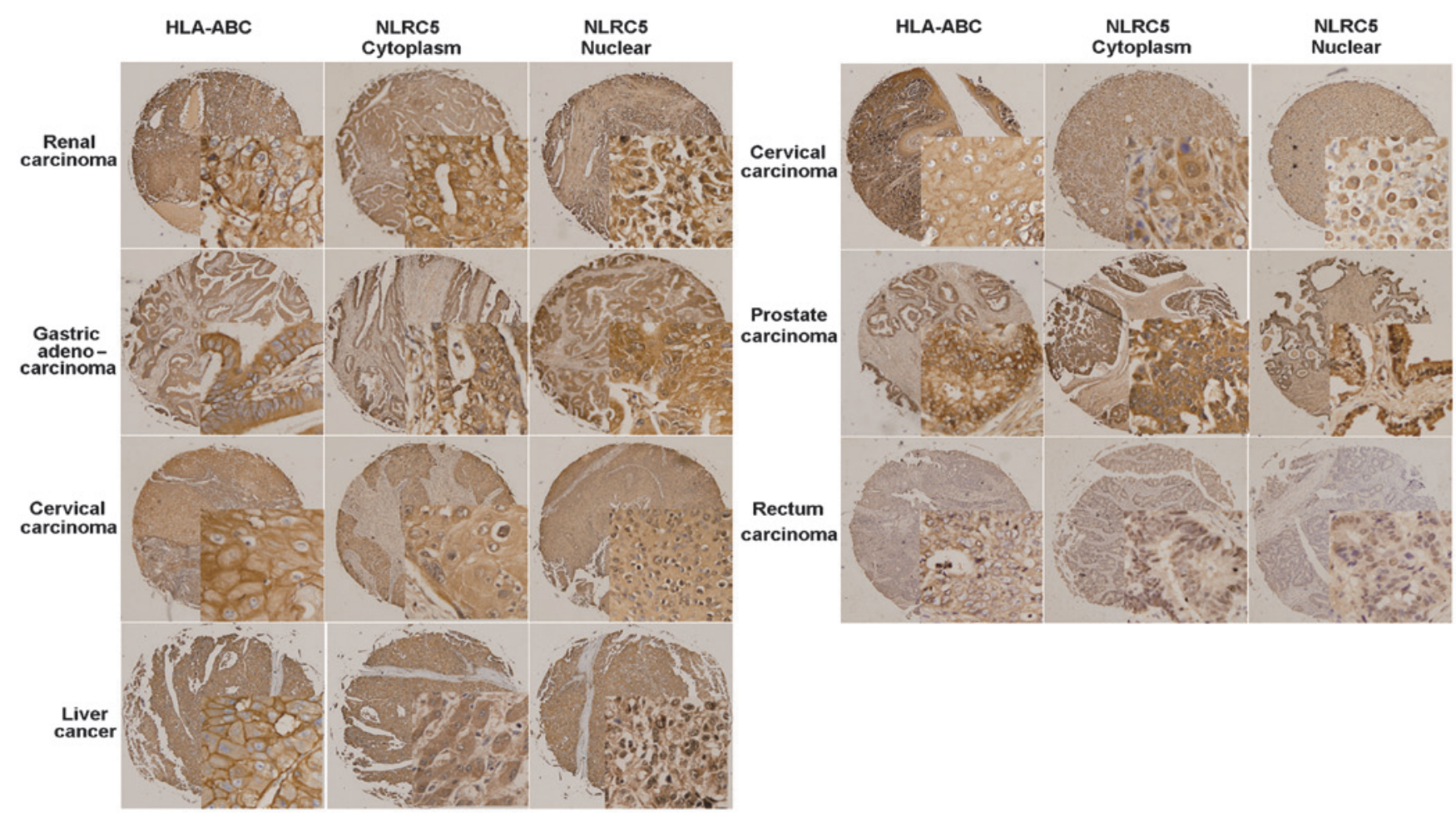

Figure 3. Immunohistochemical staining of seven human solid tumor tissue microarrays for HLA-ABC, cytoplasmic NLRC5, nuclear NLRC5. Each panel shows a representative example for the tumor tissue scored for staining intensity as positive. HLA-ABC, human leukocyte antigen-ABC; NLRC5, nucleotide-binding oligomerization-like receptor family caspase recruitment domain-containing 5.

tumor tissues and its association with the clinical outcomes of NSCLC stage III patients. The results revealed that NLRC5 and MHC class I expression were detected in NSCLC and seven other types of common human tumor tissues. IHC staining demonstrated that NLRC5 was expressed in the nucleus and cytoplasm of all eight common tumor tissues. In addition, the expression of MHC class I was found to be associated with nuclear NLRC5 in all 385 tumor cases. These results indicated that as in normal immune situations, NLRC5 regulates MHC class I expression in human tumors. By analyzing the clinical data of 62 postoperative NSCLC stage III patients, it was determined that MHC class I and nuclear NLRC5 expression were independent predictors of poor survival, which was consistent with certain previous studies $(7,21,22)$.

In the present study, there was a high level of consistency among the 62 NSCLC patients, as they all underwent radical surgery and were diagnosed with stage III-N $\mathrm{N}_{2}$ NSCLC. This may reduce the effects of difference between various tumor stages and increase credibility of the current results. In the present study, the individual IHC results for the NSCLC, liver cancer, gastric adenocarcinoma, rectal cancer, malignant melanoma tissues all revealed a correlation between the expression of MHC class I and nuclear NLRC5. However, in the renal and cervical carcinoma cases, there was no significant correlation between MHC class I and NLRC5 (cytoplasmic and nuclear) expression. This may be due to the limited number of cases for cervical cancer. While in renal carcinoma, the majority of the tumor is clear cell carcinoma, which is adenocarcinoma of the renal tubular epithelial cell; therefore, the tissue heterogeneity was different from the other tumor types examined.
Due to the role of MHC class I molecules in tumor immune surveillance and immune evasion, a number of studies have aimed to elucidate the mechanisms by which this proceeds and its association with patient prognosis $(2,23)$. Previous studies have suggested that high levels of MHC class I expression makes tumor cells promising targets for T cells; by contrast, weak expression prevents specific $\mathrm{T}$ cell recognition and results in an increased risk of disease recurrence $(3,24)$. For colorectal cancer, Simpson et al (5) reported a mean survival advantage of 26.1 months in patients whose tumors had strong MHC class I expression over those who exhibited weak MHC class I expression. Nagata et al demonstrated that HLA class I loss was associated with recurrence-free survival time, but not OS in NSCLC (25). However, certain studies reached different conclusions. Madjd et al (7) reported that the total loss of MHC class I was an independent indicator of positive prognosis in breast cancer. In addition, Ramnath et al (8) suggested that HLA class I antigen downregulation was associated with improved survival (8). In certain other types of tumors, the results also varied (26-30). MHC class I loss may affect antigen presentation to T-lymphocytes and CTL function; however, this may increase the susceptibility of tumors to NK cells and result in an improved prognostic outcome. Ramnath et al (8) indicated that there was a selective loss of MHC class I heavy chain, which was associated with improved prognosis. This may be the result of immune surveillance, which in certain patients may select against the more aggressive tumors, allowing for the growth of the more indolent HLA-negative tumors. Tumor growth may then be further controlled by NK cells (8). These previous results demonstrated that in different tumor types, $\mathrm{T}$ cell recognition 
and NK cell function were not identical and may therefore have various effects on tumor growth and patient survival.

The results of previous studies regarding MHC class I as a prognostic indicator have been controversial; this may be due to the types of samples included these studies. Certain studies have analyzed tumor patients at different disease stages with short clinical follow up $(7,8,25)$. Analysis of various tumor stages in a small group of patients may not be sufficient to determine whether MHC class I may be used as a prognostic indicator. The present study included 62 highly consistent cases of NSCLC stage III patients; therefore, these results may be more representative and indicate a novel method for predicting the prognosis of NSCLC patients. Further studies are required, with large-sample randomized experiments in order to confirm the potential of MHC class I and nuclear NLRC5 as prognostic factors for different types of cancer.

The function of NLRC5 as a MHC class I transactivator has been studied thoroughly over the past few decades. NLRC5 moves between the cytoplasm and nucleus, which indicates that it may have a nuclear function (31). The expression of NLRC5-mediated MHC class I gene requires an intact nuclear localization signal and nuclear distribution (31). Therefore, altered cellular localization of NLRC5 may impact MHC class I expression as well as MHC class I-mediated antigen presentation (9). In addition, it was reported that NLRC5 influences histone methylation (H3K27me3) and may therefore mediate gene expression through adjusting the chromosome activation status of the MHC class I locus (32). In the present study, nuclear and cytoplasmic NLRC5 expression were found to be associated with MHC class $\mathrm{I}(\mathrm{P}<0.001$ and $\mathrm{P}=0.003$, respectively).

In conclusion, the results of the present study demonstrated that NLRC5 was widely expressed in eight common human tumor tissues in the nucleus as well as the cytoplasm. Furthermore, the nuclear expression was found to be correlated with MHC class I. MHC class I heavy chain loss has been validated in a previous study to be a predictor of patient survival (22); the present study demonstrated that the expression of NLRC5 in the nucleus acted as a negative prognostic indicator in NSCLC patients. Therefore NLRC5 and MHC class I may be used in conjunction with other independent prognostic factors in order to further stratify patients for adjuvant therapy. However, in order to validate the use of these factors as cancer biomarkers to predict the patients' prognosis, randomized screening trials are required. In vitro and in vivo experiments may also be required in order to fully elucidate the effect of MHC class I and NLRC5 on the tumor growth and differentiation.

\section{Acknowledgements}

The authors would like to thank Miss. Li Li for advice and instruction in IHC and Professor Chen Xian-Cheng for assistance with microscopy and image processing. The present study was supported by a grant from the China Postdoctoral Science Foundation (grant no. 2014M552366).

\section{References}

1. Igney FH and Krammer PH: Immune escape of tumors: Apoptosis resistance and tumor counterattack. J Leukoc Biol 71: 907-920, 2002
2. Garcia-Lora A, Algarra I and Garrido F: MHC class I antigens, immune surveillance and tumor immune escape. J Cell Physiol 195: 346-355, 2003.

3. Garrido F, Cabrera T, Concha A, et al: Natural history of HLA expression during tumour development. Immunol Today 14: 491-499, 1993.

4. Kärre K: NK cells, MHC class I molecules and the missing self. Scand J Immunol 55: 221-228, 2002.

5. Simpson JA, Al-Attar A, Watson NF, Scholefield JH, Ilyas M and Durrant LG: Intratumoral T cell infiltration, MHC class I and STAT1 as biomarkers of good prognosis in colorectal cancer. Gut 59: 926-933, 2010.

6. Garcia-Lora A, Martinez M, Algarra I, Gaforio JJ and Garrido F: MHC class I-deficient metastatic tumor variants immunoselected by T lymphocytes originate from the coordinated downregulation of APM components. Int J Cancer 106: 521-527, 2003.

7. Madjd Z, Spendlove I, Pinder SE, Ellis IO and Durrant LG: Total loss of MHC class I is an independent indicator of good prognosis in breast cancer. Int J Cancer 117: 248-255, 2005

8. Ramnath N, Tan D, Li Q, et al: Is downregulation of MHC class I antigen expression in human non-small cell lung cancer associated with prolonged survival? Cancer Immunol Immunother 55: 891-899, 2006.

9. Meissner TB, Li A, Liu YJ, Gagnon E and Kobayashi KS: The nucleotide-binding domain of NLRC5 is critical for nuclear import and transactivation activity. Biochem Biophys Res Commun 418: 786-791, 2012.

10. Staehli F, Ludigs K, Heinz LX, et al: NLRC5 deficiency selectively impairs MHC Class I- dependent lymphocyte killing by cytotoxic T cells. J Immunol 188: 3820-3828, 2012.

11. Magalhaes JG, Sorbara MT, Girardin SE and Philpott DJ: What is new with Nods? Curr Opin Immunol 23: 29-34, 2011.

12. Barker BR, Taxman DJ and Ting JP: Cross-regulation between the IL-1 $\beta /$ IL-18 processing inflammasome and other inflammatory cytokines. Curr Opin Immunol 23: 591-597, 2011

13. Benko S, Magalhaes JG, Philpott DJ and Girardin SE: NLRC5 limits the activation of inflammatory pathways. J Immunol 185: 1681-1691, 2010.

14. Davis BK, Roberts RA, Huang MT, et al: Cutting Edge: NLRC5-dependent activation of the inflammasome. J Immunol 186: 1333-1337, 2011.

15. Cui J, Zhu L, Xia X, et al: NLRC5 negatively regulates the $\mathrm{NF}-\kappa \mathrm{B}$ and type I interferon signaling pathways. Cell 141: 483-496, 2010

16. Neerincx A, Lautz K, Menning M, et al: A role for the human nucleotide-binding domain, leucine-rich repeat-containing family member NLRC5 in antiviral responses. J Biol Chem 285: 26223-26232, 2010.

17. Yao Y and Qian Y: Expression regulation and function of NLRC5. Protein Cell 4: 168-175, 2013.

18. Biswas A, Meissner TB, Kawai T and Kobayashi KS: Cutting edge: Impaired MHC class I expression in mice deficient for Nlrc5/Class I transactivator. J Immunol 189: 516-520, 2012.

19. Brambilla E, Travis WD, Colby TV, et al: The new World Health Organization classification of lung tumours. Eur Respir J 18: 10591068,2001

20. Fan L, Li P, Yin Z, et al: Ribosomal s6 protein kinase 4: A prognostic factor for renal cell carcinoma. Br J Cancer 109: 1137-1146, 2013.

21. Rimsza LM, Roberts RA, Miller TP, et al: Loss of MHC class II gene and protein expression in diffuse large B-cell lymphoma is related to decreased tumor immunosurveillance and poor patient survival regardless of other prognostic factors: A follow-up study from the Leukemia and Lymphoma Molecular Profiling Project. Blood 103: 4251-4258, 2004.

22. Bijen CB, Bantema-Joppe EJ, de Jong RA, et al: The prognostic role of classical and nonclassical MHC class I expression in endometrial cancer. Int J Cancer 126: 1417-1427, 2010.

23. Hicklin DJ, Marincola FM and Ferrone S: HLA class I antigen downregulation in human cancers: T-cell immunotherapy revives an old story. Mol Med Today 5: 178-186, 1999.

24. Dunn GP, Old LJ and Schreiber RD: The three Es of cancer immunoediting. Annu Rev Immunol 22: 329-360, 2004.

25. Nagata Y, Hanagiri T, Mizukami M, et al: Clinical significance of HLA class I alleles on postoperative prognosis of lung cancer patients in Japan. Lung Cancer 65: 91-97, 2009.

26. Nijman HW, van Diest PJ, Poort-Keesom RJ, et al: T cell infiltration and MHC I and II expression in the presence of tumor antigens: An immunohistochemical study in patients with serous epithelial ovarian cancer. Eur J Obstet Gynecol Reprod Biol 94: 114-120, 2001. 
27. Cromme FV, van Bommel PF, Walboomers JM, Gallee MP, Stern PL, Kenemans P, Helmerhorst TJ, Stukart MJ and Meijer CJ: Differences in MHC and TAP-1 expression in cervical cancer lymph node metastases as compared with the primary tumours. $\mathrm{Br}$ J Cancer 69: 1176-1181, 1994

28. Cromme FV, Meijer CJ, Snijders PJ, Uyterlinde A, Kenemans P, Helmerhorst T, Stern PL, van den Brule AJ and Walboomers JM: Analysis of MHC class I and II expression in relation to presence of HPV genotypes in premalignant and malignant cervical lesions. $\mathrm{Br}$ J Cancer 67: 1372-1380, 1993.

29. Atkins D, Ferrone S, Schmahl GE, Störkel S and Seliger B: Down-regulation of HLA class I antigen processing molecules: An immune escape mechanism of renal cell carcinoma? J Urol 171: 885-889, 2004
30. Wu JD, Higgins LM, Steinle A, Cosman D, Haugk K and Plymate SR: Prevalent expression of the immunostimulatory MHC class I chain-related molecule is counteracted by shedding in prostate cancer. J Clin Invest 114: 560-568, 2004. 31. Meissner TB, Li A, Biswas A, Lee KH, Liu YJ, Bayir E, Iliopoulos D, van den Elsen PJ and Kobayashi KS: NLR family member NLRC5 is a transcriptional regulator of MHC class I genes. Proc Natl Acad Sci USA 107: 13794-13799, 2010.

32. Meissner TB, Liu YJ, Lee KH, Li A, Biswas A, van Eggermond MC, van den Elsen PJ and Kobayashi KS: NLRC5 cooperates with the RFX transcription factor complex to induce MHC class I gene expression. J Immunol 188: 4951-4958, 2012. 